\title{
Heated Allergens and Induction of Tolerance in Food Allergic Children
}

\author{
Merryn Netting ${ }^{1}$, Maria Makrides ${ }^{1}$, Michael Gold ${ }^{2}$, Patrick Quinn ${ }^{2}$ and Irmeli Penttila ${ }^{1, *}$ \\ 1 Women's and Children's Health Research Institute, University of Adelaide, 72 King William Road, \\ North Adelaide, SA 5006, Australia; E-Mails: merryn.netting@adelaide.edu.au (M.N.); \\ maria.makrides@adelaide.edu.au (M.M.) \\ 2 Children's Youth and Women's Health Network, University of Adelaide, 72 King William Road, \\ North Adelaide, SA 5006, Australia; E-Mails: michael.gold@adelaide.edu.au (M.G.); \\ patrick.quinn@adelaide.edu.au (P.Q.) \\ * Author to whom correspondence should be addressed; E-Mail: irmeli.penttila@adelaide.edu.au; \\ Tel.: +61-8-8161-7443; Fax: +61-8-239-0267.
}

Received: 28 March 2013; in revised form: 15 May 2013 / Accepted: 17 May 2013 /

Published: 5 June 2013

\begin{abstract}
Food allergies are one of the first manifestations of allergic disease and have been shown to significantly impact on general health perception, parental emotional distress and family activities. It is estimated that in the Western world, almost one in ten children have an IgE-mediated allergy. Cow's milk and egg allergy are common childhood allergies. Until recently, children with food allergy were advised to avoid all dietary exposure to the allergen to which they were sensitive, in the thought that consumption would exacerbate their allergy. However, recent publications indicate that up to $70 \%$ of children with egg allergy can tolerate egg baked in a cake or muffin without apparent reaction. Likewise, up to $75 \%$ of children can tolerate baked goods containing cow's milk, and these children demonstrate $\operatorname{IgE}$ and $\mathrm{IgG} 4$ profiles indicative of tolerance development. This article will review the current literature regarding the use of heated food allergens as immunotherapy for children with cow's milk and egg allergy.
\end{abstract}

Keywords: egg; milk; allergy; heated allergens; tolerance; oral; immunotherapy 


\section{Introduction}

Food allergies have become increasingly common around the world, and it is estimated that globally, about 220-520 million people may suffer from food allergy [1]. Although it is possible to develop an IgE-mediated allergy to any food, most individuals with allergies react to one or a combination of nine common foods: cow's milk, soy, egg, wheat, peanut, tree nuts, sesame, fish and shellfish [2].

Food allergies are one of the first manifestations of allergic disease and have been shown to significantly impact on general health perception, parental emotional distress and family activities [3]. Young children, in particular, are at risk of developing food allergy, and it is estimated that 5\% to $8 \%$ of toddlers are affected, compared with $1 \%$ to $2 \%$ of adults [1]. The incidence of allergic diseases is increasing globally, and this poses a major burden to healthcare costs in every country around the world [1].

Many children outgrow their food allergy; however, recent data suggest that the natural history of food allergy has changed, and it is now common for allergies to persist to late childhood and early adulthood $[4,5]$.

In an affected patient with a food allergy, management consists of removal of the food from the diet and provision of an action plan for management of any accidental exposure to the food protein. More recently, complete exclusion of the food from a food allergic individual has been questioned. It is possible that including the allergenic food in the diet has potential to increase the likelihood of a child outgrowing the allergy more quickly or to allow a certain amount of the food to be tolerated [6]. Complete and absolute avoidance of a food allergen may not be necessary in a child's diet if they show tolerance to that food in an alternative format. Many children with cow's milk or egg allergies tolerate foods containing baked milk [7] or egg protein [8], and the hypothesis that this might be associated with a move towards tolerance of the protein has moved dietary management strategies away from strict avoidance to allowing the inclusion of the baked protein in the diet if it is tolerated [9-11].

With the rising incidence in food allergies there has been interest in improving their management, with the aim of offering a treatment strategy that does not solely rely on avoidance of food proteins in the diet. Immunotherapy is used successfully for managing venom and pollen allergies, and thus, there has been increasing research into the use of immunotherapy to manage common food allergies [12]. This review will summarize current research related to oral immunotherapy for management of IgE-mediated cow's milk and egg allergy and, in particular, the potential for heated proteins to be used as vehicles for oral immunotherapy.

\section{Development of Oral Tolerance}

The intestine is the largest immune organ in the body, and it works to protect the body from a constant onslaught of pathogens and ingested proteins derived from the outside environment by secreting Immunoglobulin A (IgA) and activating immune cells to fight pathogens or to act as regulatory cells to maintain gut homeostasis. Gut-associated lymphoid tissue (GALT), in particular, Peyer's Patches, are involved in the induction of immunity and maintenance of gut homeostasis [13,14]. The gut epithelium, along with dendritic cells, plays an active role in this process by continually 
sampling the gut luminal contents [15]. The interactions between microbes, epithelium and GALT are involved in developing memory of the immune system and are essential for the development of oral tolerance [16].

Our understanding of the exact mechanisms involved in the development of tolerance to an allergen is evolving; however, the precise mechanism(s) by which the normal intestinal immune system responds to food and its involvement in the development of allergy remains essentially unresolved [17,18]. Development of oral tolerance is dependent on a number of early events, including allergen exposure (age, dose and timing), gut colonisation and the influence of maternal milk [19-25]. The resulting regulated immune response is an antigen driven process involving repeated exposure to the antigen and establishment of healthy gut colonization with commensal bacteria [25].

Development of oral tolerance to a food allergen involves early changes at the level of the gut mucosa [26]. Initially, there is a need to establish a local immunosuppressive gut milieu dominated by immunomodulatory cytokines, such as IL-10 and TGF $\beta$, which control inflammation, non-specifically [27]. This environment is then conducive to the development of a highly regulated systemic response and differentiation of antigen-specific $\left(\mathrm{CD} 4{ }^{+} \mathrm{CD} 25^{+} \mathrm{FOXP} 3^{+}\right)$regulatory $\mathrm{T}$-cells (T-regs), essential for immune homeostasis in the intestine [23,24,28-31]. However, the role of these regulatory $\mathrm{T}$-cells in food allergic disease and regulation of immune response development to allergens in the gut remains unclear. Studies have shown that transcription factor Foxp3 is critical for the development and function of T-regs. A variant of IPEX syndrome, which is characterised by autoimmune and severe allergic symptoms, including severe enteropathy, food allergies, atopic dermatitis, hyper-IgE and eosinophilia, is associated with a mutation within an upstream non-coding region of Foxp3 that affects its function [32]. Recent studies have also shown that in the intestine retinoic acid producing dendritic cells and Foxp $3^{+}$T-regs interact to induce oral tolerance $[33,34]$. With regard to food allergy, the frequency of Foxp $3^{+}$cells in the periphery is low in individuals with food allergies, and children with food allergy have reduced T-regulatory cell function [35]. Other studies have shown that a higher frequency of food allergen-specific T-regulatory cells correlates with a milder clinical phenotype and favourable prognosis [36].

\section{Immunotherapy for Food Allergies}

Given the potential of exposure to a small amount of allergen to promote tolerance to that allergen and the successful use of immunotherapy for management of venom and pollen allergies, there has been increasing research into the use of immunotherapy to manage common food allergies [12]. Early use of subcutaneous immunotherapy for food allergy resulted in severe reactions, and so, recent focus has been on oral immunotherapy [37]. Forms of oral immunotherapy to treat food allergy include specific oral tolerance induction (SOTI) [12], where the allergen is ingested, or sublingual immunotherapy (SLIT), where a minute amount (picograms) of allergen, in the form of allergen extract or diluted allergen, is placed under the tongue [12,38]. Both approaches start with a 'build up phase', initially starting with very small, diluted doses of the food protein with the amount of protein included in the dose slowly increased in a stepwise manner. This is followed by a "maintenance phase" with a consistent dose of allergen given at regular intervals. The regimes are followed with a "discontinuation phase" and a "tolerance food challenge" to test the effectiveness of the 
immunotherapy treatment. The entire process may take 12 months or longer to complete. Adverse events are common during oral immunotherapy - this risk of an adverse event increases with illness, exercise, menses and every time the dose of the food is increased [39,40]. If regular consumption of the allergen is discontinued during the maintenance phase, there is a risk that tolerance will be broken, and an allergic reaction may follow exposure [38]. For food allergy, the endpoint of immunotherapy may not always be tolerance - the therapy may result in desensitization, an increase in the antigen dose required to elicit symptoms arising from a transient altered immune response and dependent on regular (daily) exposure to the allergen, or the therapy may result in true tolerance [41] - the ability to ingest the allergen without any symptoms once regular exposure has ceased, owing to persistent changes in the immune response. Desensitization still has a benefit in that larger doses of the allergen can be tolerated before eliciting a hypersensitivity reaction, thus lessening the potential for an anaphylactic reaction from accidental exposure.

Oral immunotherapy has been attempted for many food proteins, but we have specifically chosen to discuss cow's milk and egg oral immunotherapy, because of the interest in using baked forms of these foods as a treatment strategy. There is one Cochrane review from 2012 [42] on oral immunotherapy for cow's milk allergy, but none for egg. The Cochrane review included 16 studies, representing five trials and concluded that milk oral immunotherapy can lead to desensitization in the majority of individuals, although development of long term-tolerance has not been established.

Key trials reporting cow's milk oral immunotherapy and egg oral immunotherapy in the last 10 years are summarized in Tables 1 and 2. Nine studies reported outcomes of cow's milk oral immunotherapy and also nine for egg oral immunotherapy. These include three randomized controlled trials (RCTs) for cow's milk SOTI [38,43,44] and three RCTs investigating egg oral immunotherapy [45-47], but most of the studies report on open, uncontrolled prospective trials. We have added one trial [38] that was not included in the cows' milk immunotherapy Cochrane review. These studies have limitations. The studies utilized highly variable selection criteria, dosing regimens and maintenance doses, making them difficult to compare. The individual studies reported have limited statistical power, as only a small number of children were included in the treatment group.

All of the 18 studies reported SOTI, and one study reported results of a SLIT/SOTI combination protocol for cow's milk immunotherapy. Keet et al. [38] compared the outcome of children with cow's milk allergy who were given a SLIT protocol initially and then randomized to a low or high dose SOTI protocol or SLIT alone. More children in the SLIT followed by the high dose SOTI group passed the final oral food challenge after six weeks compared to those in SLIT alone or the lower SOTI dose.

Dosing regimens varied from $15 \mathrm{~mL}$ to $200 \mathrm{~mL}$ cow's milk and $0.3 \mathrm{~g}$ to $60 \mathrm{~g}$ of egg for the maintenance phase. Furthermore the allergen used for the reported regimes varied - for example fresh raw whole egg, fresh raw egg white, pasteurized egg white, pasteurized whole egg, lightly cooked egg have all been used for the egg oral immunotherapy studies. Most of the cow's milk immunotherapy studies used fresh cow's milk, but some reported use of dried non fat milk powder. Some of the studies asked participants to consume the maintenance dose on a daily basis, and some required the dose to be consumed every 2-3 days. 
Table 1. Summary of key egg-specific oral immunotherapy trials.

\begin{tabular}{|c|c|c|c|c|c|c|}
\hline Study & Study Design & $\begin{array}{l}\text { Subjects } \\
N \text {, age (range) } \\
\text { in active groups }\end{array}$ & Protocol & Clinical Outcomes & $\begin{array}{l}\text { Clinical Results: } \\
\text { if no endpoint challenge, } \\
\text { tolerance was determined by } \\
\text { tolerance of maintenance dose }\end{array}$ & Immunological Outcomes \\
\hline Burks, 2012 [46] & RCT & $\begin{array}{l}N=40 \\
7 \text { years } \\
(5-11)\end{array}$ & $\begin{array}{l}\text { SOTI } \\
\text { Egg white powder } v s . \\
\text { corn starch } \\
\text { Blinded until } 10 \text { month } \\
\text { challenge }\end{array}$ & $\begin{array}{l}\text { Maintenance: up to } 2 \mathrm{~g} \text { egg } \\
\text { white powder daily ( } 1 / 3 \mathrm{egg}) \\
\text { Endpoint challenge: } \\
\text { OFC at } 10 \text { and } 22 \text { months ( } 5 \mathrm{~g} \\
\text { EW powder) } \\
\text { If child passed } 22 \text { month OFC, } \\
\text { OIT ceased and egg-free diet } \\
\text { for } 4-6 \text { weeks. At } 24 \text { months, } \\
\text { these children had an OFC with } \\
10 \mathrm{~g} \text { egg white powder and one } \\
\text { cooked egg. } \\
\text { Evaluations at } 30 \text { and } \\
36 \text { months per telephone. }\end{array}$ & $\begin{array}{l}\text { At } 10 \text { month OFC: } \\
22 / 35 \text { tolerant } \\
1 / 35 \text { withdrew } \\
\text { At } 22 \text { month } \text { OFC } \\
30 / 34 \text { tolerant } \\
\text { At } 24 \text { months } \text { OFC: } \\
11 / 29 \text { tolerant } \\
\mathbf{3 0} \text { months phone call: } \\
11 / 11 \text { tolerant } \\
\mathbf{3 6} \text { months phone call: } \\
10 / 10 \text { tolerant }\end{array}$ & $\begin{array}{l}\text { SPT } \downarrow \\
\operatorname{IgE} \downarrow \\
\operatorname{IgG} \uparrow \\
\text { Egg-specific basophil activation } \downarrow\end{array}$ \\
\hline Meglio 2013 [45] & RCT & $\begin{array}{l}N=10 \\
\text { median age } \\
8.4 \text { years }\end{array}$ & $\begin{array}{l}\text { SOTI } \\
\text { Adjunct Therapy } \\
\text { Raw fresh egg } v s . \\
\text { egg-free diet }\end{array}$ & $\begin{array}{l}\text { Maintenance: } \\
25 \mathrm{~mL} \text { raw egg } \\
\text { three-times/week, alternatively } \\
\text { could consume foods } \\
\text { corresponding to about three } \\
\text { eggs per week. } \\
\text { Endpoint challenge: } \text { No } \\
\end{array}$ & $\begin{array}{l}\text { At six months: } \\
8 / 10 \text { tolerant } \\
1 / 10 \text { partially tolerant } \\
1 / 10 \text { withdrew } \\
\text { ( } 2 / 10 \text { in control group achieved } \\
\text { tolerance of raw egg in same time } \\
\text { period.) }\end{array}$ & $\begin{array}{l}\text { SPT } \downarrow \\
\text { SPT } \downarrow \\
\operatorname{IgG} 4 \text { ovomucoid } \leftrightarrow \\
\operatorname{IgG} 4 \text { ovalbumin } \uparrow \\
\text { Cytokines and transforming growth factors (IL-5 } \uparrow \text { ) }\end{array}$ \\
\hline $\begin{array}{l}\text { Dello Iacono } 2013 \\
\text { [47] }\end{array}$ & $\mathrm{RCT}$ & $\begin{array}{l}N=10 \\
7 \text { years, } 7 \text { months } \\
(5-11)\end{array}$ & $\begin{array}{l}\text { SOTI } \\
\text { Raw fresh egg vs. egg } \\
\text { free diet }\end{array}$ & $\begin{array}{l}\text { Maintenance: } \\
40 \mathrm{~mL} \text { raw egg (one small egg). } \\
\text { Endpoint challenge: No }\end{array}$ & $\begin{array}{l}\text { At six months: } \\
9 / 10 \text { partially tolerant } \\
1 / 10 \text { no tolerance }\end{array}$ & $\begin{array}{l}\text { SPT } \downarrow \\
\operatorname{IgE} \downarrow\end{array}$ \\
\hline $\begin{array}{l}\text { Patriarca, et al. } \\
2007 \text { [48] }\end{array}$ & $\begin{array}{l}\text { Prospective, } \\
\text { open study } \\
\text { with reference } \\
\text { group }\end{array}$ & $\begin{array}{l}N=14 \\
3-16 \text { years }\end{array}$ & $\begin{array}{l}\text { Sublingual initially, then } \\
\text { higher doses orally. } \\
\text { Adjunct therapy } \\
\text { Raw fresh egg }\end{array}$ & $\begin{array}{l}\text { Maintenance: one egg } \\
2-3 \text { times/week. } \\
\text { Endpoint challenge: No }\end{array}$ & $\begin{array}{l}10 / 14 \text { tolerant } \\
1 / 14 \text { partially tolerant } \\
1 / 14 \text { failed } \\
2 / 14 \text { withdrew }\end{array}$ & $\begin{array}{l}\text { SPT } \downarrow \\
\operatorname{IgE} \downarrow \\
\operatorname{IgG} 4 \uparrow\end{array}$ \\
\hline
\end{tabular}


Table 1. Cont.

\begin{tabular}{|c|c|c|c|c|c|c|}
\hline $\begin{array}{l}\text { Fuentes-Aparico } \\
2012 \text { [49] }\end{array}$ & $\begin{array}{l}\text { Prospective, } \\
\text { open study } \\
\text { with reference } \\
\text { group }\end{array}$ & $\begin{array}{l}N=19 \\
\text { mean } 9.2 \text { years } \\
(4-14)\end{array}$ & $\begin{array}{l}\text { SOTI } \\
\text { Pasteurised egg powder }\end{array}$ & $\begin{array}{l}\text { Maintenance: } \\
10 \mathrm{~g} \text { powdered egg (equivalent to } \\
\text { one egg) } \\
\text { Endpoint challenge: No } \\
\text { Follow up at six and } 12 \text { months }\end{array}$ & $\begin{array}{l}16 / 19 \text { tolerant } \\
2 / 19 \text { withdrew } \\
1 / 19 \text { partially tolerant to cooked } \\
\text { egg }\end{array}$ & $\begin{array}{l}\text { IgE } \leftrightarrow \\
\text { IgG4 } \uparrow \\
\text { T-cell subtypes: } \\
\text { B-cells, NK cells, NKT cells, CD8+T-cells: } \leftrightarrow \\
\text { Effector memory CD4+T-cells (TEM): } \downarrow \\
\text { CD4+ recent thymic emigrants (RTEs): } \uparrow \\
\text { CD38/RO neg T-cells: } \uparrow \\
\text { Cytokines: IL-12, IL4, IL-13 \& IL- } \beta: \leftrightarrow \\
\text { Th1 cytokines (IL-2, TNF- } \alpha \text { and IFN } \gamma \text { ): } \downarrow \\
\text { Th2 cytokines ( IL-5 and IL-10) \& Th9 (IL-9), Th22 } \\
\text { (IL-22) and Th17 cytokines (IL-17A): } \downarrow \\
\text { IL-13 } \uparrow(\text { trend NS) }\end{array}$ \\
\hline $\begin{array}{l}\text { Patriarca et al. } \\
2003[50]\end{array}$ & $\begin{array}{l}\text { Prospective, } \\
\text { open study }\end{array}$ & $\begin{array}{l}N=12 \\
<16 \text { years }\end{array}$ & $\begin{array}{l}\text { SOTI } \\
\text { Adjunct therapy } \\
\text { Raw fresh egg }\end{array}$ & $\begin{array}{l}\text { Maintenance: one egg }(50 \mathrm{~mL}) \\
2-3 \text { times/week. } \\
\text { 3-8 months } \\
\text { Endpoint challenge: No }\end{array}$ & $\begin{array}{l}10 / 12 \text { tolerant } \\
2 / 12 \text { withdrew }\end{array}$ & $\begin{array}{l}\mathrm{SPT} \downarrow \leftrightarrow \\
\mathrm{IgE} \downarrow \\
\mathrm{IgG} 4 \uparrow\end{array}$ \\
\hline $\begin{array}{l}\text { Buchanan et al. } \\
2007 \text { [51] }\end{array}$ & $\begin{array}{l}\text { Prospective, } \\
\text { open study }\end{array}$ & $\begin{array}{l}N=7,4 \text { years } \\
(14-84 \text { months })\end{array}$ & $\begin{array}{l}\text { SOTI } \\
\text { Egg white powder }\end{array}$ & $\begin{array}{l}\text { Maintenance: } 300 \mathrm{mg} \text { daily for } \\
24 \text { months. } \\
\text { Endpoint challenge: } \\
\text { DBPCFC at } 24 \text { months ( } 10 \mathrm{~g} \text { egg } \\
\text { white). If passed, followed by } \\
\text { open scrambled egg challenge. }\end{array}$ & $\begin{array}{l}24 \text { month DBPCFC } \\
4 / 7 \text { tolerant. } \\
\text { 3/7 failed, but tolerated } \\
\text { more egg }\end{array}$ & $\begin{array}{l}\operatorname{IgE} \downarrow(\mathrm{NS}) \\
\mathrm{IgG} 4 \uparrow\end{array}$ \\
\hline Itoh, 2010 [52] & $\begin{array}{l}\text { Prospective, } \\
\text { open study }\end{array}$ & $\begin{array}{l}N=6 \\
\text { mean } 9.7 \text { years } \\
(7-12)\end{array}$ & $\begin{array}{l}\text { SOTI } \\
\text { Raw powdered egg white } \\
\text { and lightly cooked } \\
\text { (whole) egg }\end{array}$ & $\begin{array}{l}\text { Maintenance: one whole egg } \\
(60 \mathrm{~g}) \text { At least two times/week. } \\
\text { Endpoint challenge: } \\
\text { OFC to powdered egg at } \\
9-12 \text { months after starting } \\
\text { maintenance. }\end{array}$ & $\begin{array}{l}\text { 9-12 months } \mathrm{OFC} \text { : } \\
\text { 3/6 tolerant } \\
\text { 3/6 failed } \\
\text { At } 16 \text { months: } \\
6 / 6 \text { tolerated }>60 \text { g heated egg. }\end{array}$ & $\begin{array}{l}\text { SPT } \leftrightarrow \\
\operatorname{IgE} \uparrow \text { then } \downarrow \\
\operatorname{IgG} 4 \uparrow \\
\text { Histamine release test } \downarrow ; \\
\text { Th1/Th2 ratio } \downarrow \text { at six months, not at } 12 \text { months; } \\
\text { IL- } 4 \text {, IFN } \gamma \leftrightarrow ; \text { IL-10 } \downarrow, \text { TGF } 1 \uparrow\end{array}$ \\
\hline $\begin{array}{l}\text { Garcia } \\
\text { Rodriguez, 2011 } \\
\text { [53] } \\
\text { Urra, 2012 [54] }\end{array}$ & $\begin{array}{l}\text { Prospective, } \\
\text { open study }\end{array}$ & $\begin{array}{l}N=23 \\
\text { mean } 8.1 \text { years } \\
(5-17)\end{array}$ & $\begin{array}{l}\text { SOTI } \\
\text { Pasteurised raw egg white } \\
\text { and whole cooked egg }\end{array}$ & $\begin{array}{l}\text { Maintenance: one cooked egg } \\
\text { daily for three months. } \\
\text { Exposure then extended to once in } \\
48 \mathrm{~h} \text { and, at six months once, in } \\
72 \mathrm{~h} . \\
\text { Endpoint challenge: No }\end{array}$ & $\begin{array}{l}\text { 20/23 tolerant } \\
1 / 23 \text { withdrew } \\
2 / 23 \text { switched to slow protocol } \\
\text { and achieved tolerance. }\end{array}$ & $\begin{array}{l}\text { SPT } \downarrow \\
\text { IgE } \downarrow \\
\text { IgG4 } \uparrow \\
\text { CD4 + FoxP3 + cells: } \uparrow\end{array}$ \\
\hline
\end{tabular}

N, number; RCT, randomized controlled trial; SOTI, specific oral tolerance induction; OFC, Oral Food Challenge; DBPCFC, Double Blind, Placebo Controlled Food Challenge; EPSPT, Endpoint titration skin prick testing; SPT, skin prick test; NS, not significant. 
Table 2. Summary of key cow's milk specific oral immunotherapy trials.

\begin{tabular}{|c|c|c|c|c|c|c|}
\hline $\begin{array}{l}\text { Study and } \\
\text { Study Type }\end{array}$ & Study Design & $\begin{array}{l}\text { Subjects } \\
N \text {, age (range) } \\
\text { in active groups }\end{array}$ & Protocol & Outcomes & $\begin{array}{l}\text { Clinical Results: } \\
\text { if no endpoint challenge, } \\
\text { tolerance was determined by } \\
\text { tolerance of maintenance dose. }\end{array}$ & Immunological Outcomes \\
\hline $\begin{array}{l}\text { Skripak } 2008 \\
{[43]}\end{array}$ & $\mathrm{RCT}$ & $\begin{array}{l}N=13 \\
9 \text { years }(6-21)\end{array}$ & $\begin{array}{l}\text { SOTI } \\
\text { Dried powdered milk } v s . \\
\text { Profree }\end{array}$ & $\begin{array}{l}\text { Maintenance: } 500 \mathrm{mg} \text { milk } \\
\text { protein }(15 \mathrm{~mL} \text { milk }) \\
\text { Endpoint challenge: } \mathrm{DBPCFC} \\
\text { up to } 2000 \mathrm{mg} \text {, cumulative dose } \\
\text { of } 8 \mathrm{~g} \text { milk protein }\end{array}$ & $\begin{array}{l}12 / 13 \text { tolerant } \\
1 / 13 \text { withdrew }\end{array}$ & $\begin{array}{l}\operatorname{IgE} \uparrow \\
\operatorname{IgG} 4 \uparrow\end{array}$ \\
\hline $\begin{array}{l}\text { Martorell } \\
2011[44]\end{array}$ & $\mathrm{RCT}$ & $\begin{array}{l}N=30 * \\
2 \text { years }(2-3)\end{array}$ & $\begin{array}{l}\text { SOTI } \\
\text { Fresh cow's milk vs. milk- } \\
\text { free diet }\end{array}$ & $\begin{array}{l}\text { Maintenance: } 200 \mathrm{~mL} \text { daily } \\
\text { plus unlimited dairy products. } \\
\text { Endpoint challenge: only in two } \\
\text { children who did not } \\
\text { tolerate SOTI. }\end{array}$ & $\begin{array}{l}\text { At } 16 \text { weeks: } \\
27 / 30 \text { tolerant } \\
1 / 30 \text { partially tolerant } \\
1 / 30 \text { - no tolerance } \\
1 / 30 \text { - withdrew }\end{array}$ & $\operatorname{IgE} \downarrow$ \\
\hline Keet 2012 [38] & $\mathrm{RCT}$ & $\begin{array}{l}N=30 \\
6-16 \text { years } \\
\text { SLIT group } n=10 \\
\text { median age: } 8 \text { year } \\
(6-11), \\
\text { OITB group } n=10 \\
9 \text { year }(6-15), \\
\text { OITA group } n=10 \\
8 \text { years }(6-16)\end{array}$ & $\begin{array}{l}\text { SLIT alone, vs. SLIT } \\
\text { followed by SOTI (high or } \\
\text { low dose). } \\
\text { SLIT, target dose } 7 \mathrm{mg} \text {. } \\
\text { OITA, target dose of } 2 \mathrm{~g} \text {. } \\
\text { OITB, target dose of } 1 \mathrm{~g} \text {. } \\
\text { SLIT, CM allergenic extract } \\
\text { SOTI, dried non-fat milk } \\
\text { powder }\end{array}$ & $\begin{array}{l}\text { Maintenance: } \\
\text { SLIT, target dose } 7 \mathrm{mg} . \\
\text { OITA }=\text { target dose of } 2 \mathrm{~g} . \\
\text { OITB }=\text { target dose of } 1 \mathrm{~g} . \\
\text { Endpoint challenge: OFC to } 8 \mathrm{~g} \\
\text { cow's milk protein at } 32 \text { and } \\
80 \text { weeks (on therapy) and at } \\
81 \text { and } 86 \text { weeks (off therapy) }\end{array}$ & $\begin{array}{l}\text { 28/30 completed protocol } \\
\text { 2/30 withdrew } \\
\text { Overall at end of protocol } \\
\text { SLIT/SLIT: } 1 / 10 \text { tolerant } \\
\text { SLIT/OITB: } 3 / 10 \text { tolerant } \\
\text { SLIT/OITA: } 5 / 10 \text { tolerant } \\
\text { Challenge threshold at } 32 \text { weeks } \\
\text { (12 weeks of maintenance) } \\
\text { SLIT: } 7 \times \text { increase } \\
\text { OITB: } 64 \times \text { increase } \\
\text { OITA: } 79 \times \text { increase } \\
\text { Desensitization } v \text { s. tolerance: } \\
\mathbf{8 1} \text { week challenge (one week off } \\
\text { therapy): } \\
\text { 2/10 (SLIT/OITB group) failed, } \\
\text { both requiring adrenalin. } \\
\mathbf{8 6} \text { week challenge: } \\
\text { SLIT/OITB group } 1 / 10 \text { failed } \\
\text { SLIT/OITA group } 3 / 10 \text { failed }\end{array}$ & $\begin{array}{l}\text { EPSPT } \\
\text { IgE } \downarrow \text { (in OIT, but not SLIT) } \\
\text { IgG4 个 } \\
\text { basophil histamine release } \downarrow \text { (NS), } \\
\text { constitutive CD63 expression } \downarrow \text { (SLIT/SLIT group), } \\
\text { CD203c expression } \downarrow \text {, intracellular spleen tyrosine } \\
\text { kinase (Syk) levels } \leftrightarrow\end{array}$ \\
\hline
\end{tabular}


Table 2. Cont.

\begin{tabular}{|c|c|c|c|c|c|c|}
\hline $\begin{array}{l}\text { Patriarca } 2003 \\
{[50]}\end{array}$ & $\begin{array}{l}\text { Prospective, } \\
\text { open study with } \\
\text { reference group }\end{array}$ & $\begin{array}{l}N=16 \\
<16 \text { years }\end{array}$ & $\begin{array}{l}\text { SOTI } \\
\text { adjunct therapy } \\
\text { fresh cow's milk }\end{array}$ & $\begin{array}{l}\text { Maintenance: } 120 \mathrm{~mL} \text { milk at least } \\
2-3 \text { times/week } \\
\text { Endpoint challenge: } \text { No }\end{array}$ & $\begin{array}{l}10 / 16 \text { tolerant } \\
3 / 16 \text { interrupted schedule } \\
3 / 16 \text { withdrew } \\
\end{array}$ & $\begin{array}{l}\mathrm{SPT} \downarrow \leftrightarrow \\
\mathrm{IgE} \downarrow \\
\mathrm{IgG} 4 \uparrow\end{array}$ \\
\hline $\begin{array}{l}\text { Patriarca } 2007 \\
{[48]}\end{array}$ & $\begin{array}{l}\text { Prospective, } \\
\text { open study with } \\
\text { reference group }\end{array}$ & $\begin{array}{l}N=14 \\
3-16 \text { years }\end{array}$ & $\begin{array}{l}\text { SLIT, then SOTI. } \\
\text { adjunct therapy } \\
\text { fresh cow's milk }\end{array}$ & $\begin{array}{l}\text { Maintenance: } 130 \mathrm{~mL} \text { milk at least } \\
2-3 \text { times a week } \\
\text { Endpoint challenge: No }\end{array}$ & $\begin{array}{l}10 / 14 \text { tolerant } \\
1 / 14 \text { partially tolerant } \\
1 / 14 \text { failed } \\
2 / 14 \text { withdrew }\end{array}$ & $\begin{array}{l}\mathrm{SPT} \downarrow \\
\mathrm{IgE} \downarrow \\
\mathrm{IgG} 4 \uparrow\end{array}$ \\
\hline $\begin{array}{l}\text { Meglio 2004 [55] } \\
\text { \& } 2008[56]\end{array}$ & $\begin{array}{l}\text { Prospective, } \\
\text { open study }\end{array}$ & $\begin{array}{l}N=21 \\
6 \text { years }(5-10)\end{array}$ & $\begin{array}{l}\text { SOTI } \\
\text { Adjunct Therapy } \\
\text { Fresh cow's milk }\end{array}$ & $\begin{array}{l}\text { Maintenance: } 200 \mathrm{~mL} \text { milk per day for } \\
\text { six months } \\
\text { Endpoint challenge: No }\end{array}$ & $\begin{array}{l}15 / 21 \text { tolerant } \\
(8 / 15 \text { asymptomatic } \\
7 / 15 \text { symptomatic and managed } \\
\text { with adjunct therapy) } \\
3 / 21 \text { withdrew } \\
\text { After four years } \\
13 / 20 \text { tolerant } \\
1 / 20 \text { partially tolerant } \\
\end{array}$ & $\begin{array}{l}\text { SPT } \\
\text { EPST } \downarrow \\
\operatorname{IgE} \leftrightarrow \\
\text { Faecal blood: no occult blood reported. }\end{array}$ \\
\hline $\begin{array}{l}\text { Narisety } 2009[57] \\
\text { Follow up of [43] }\end{array}$ & $\begin{array}{l}\text { Prospective, } \\
\text { open study }\end{array}$ & $\begin{array}{l}N=15 \\
6-16 \text { years, } \\
\text { tolerating } 75 \mathrm{~mL} \\
\text { cow's milk. }\end{array}$ & $\begin{array}{l}\text { Tolerant children increased } \\
\text { dose of dried powdered milk } \\
\text { by } 50 \% \text { every two weeks. }\end{array}$ & $\begin{array}{l}\text { Maintenance: median dose } 7000 \mathrm{mg} \text { milk } \\
\text { protein }(\approx 200 \mathrm{~mL} \text { milk) } \\
\text { Endpoint challenge: } \mathrm{OFC} \text { at } 13-75 \text { weeks } \\
\text { cumulative milk dose of } 16 \mathrm{mg} \text { milk } \\
\text { protein }(480 \mathrm{~mL})\end{array}$ & $\begin{array}{l}\text { At } \mathbf{1 3}-\mathbf{7 5} \text { weeks } \\
6 / 16 \text { tolerant } \\
7 / 16 \text { partially tolerant } \\
2 / 16 \text { ongoing symptoms with } \\
\text { maintenance, so no OFC } \\
\end{array}$ & $\begin{array}{l}\text { EPSPT } \downarrow \\
\operatorname{IgE} \downarrow \\
\operatorname{IgG} 4 \uparrow\end{array}$ \\
\hline Alvaro 2012 [58] & $\begin{array}{l}\text { Prospective, } \\
\text { open study }\end{array}$ & $\begin{array}{l}N=66 \\
\text { mean } 8 \text { years } \\
(44 / 66 \text { with } \\
\text { anaphylaxis to } \\
\text { cow's milk ) }\end{array}$ & $\begin{array}{l}\text { SOTI } \\
\text { fresh cow's milk }\end{array}$ & $\begin{array}{l}\text { Maintenance: } 200 \mathrm{~mL} \text { daily } \\
\text { Endpoint challenge: No }\end{array}$ & $\begin{array}{l}\text { non anaphylactic group: } \\
\text { 16/22 tolerant } \\
6 / 22 \text { partially tolerant } \\
\text { anaphylactic group: } \\
35 / 44 \text { tolerant. } \\
7 / 44 \text { partially tolerant } \\
1 / 44 \text { tolerant to } 1 \mathrm{~mL} \\
1 / 44 \text { withdrew } \\
\end{array}$ & $\operatorname{IgE} \downarrow$ \\
\hline Bedoret 2012 [59] & $\begin{array}{l}\text { Prospective, } \\
\text { open study }\end{array}$ & $\begin{array}{l}N=10 \\
8 \text { years }(7-17)\end{array}$ & $\begin{array}{l}\text { SOTI } \\
\text { adjunct therapy } \\
\text { fresh cow's milk }\end{array}$ & $\begin{array}{l}\text { Maintenance: } 2000 \mathrm{mg} \text { cow's milk } \\
\text { protein }(60 \mathrm{~mL}) \\
\text { Endpoint challenge: } \mathrm{DBPCFC} \text { at week } 24 \\
\text { Cumulative dose of } 7250 \mathrm{mg} \text {, then open } \\
\text { challenge of } 120-240 \mathrm{~mL} \text { milk. }\end{array}$ & $\begin{array}{l}\text { At week } 24 \\
9 / 10 \text { tolerant } \\
1 / 10 \text { withdrew }\end{array}$ & $\begin{array}{l}\text { SPT } \downarrow \\
\text { IgE } \downarrow \\
\text { IgG4 } \uparrow \\
\text { CD4 T-cell proliferation } \downarrow \\
\text { T-reg cells } \leftrightarrow \\
\text { IFN } \gamma / \text { IL-4 ratio } \uparrow \\
\text { Basophil activation } \downarrow\end{array}$ \\
\hline
\end{tabular}


Not all of the studies assessed actual tolerance to cow's milk or egg at the end of the intervention. Some used a double blinded, placebo controlled food challenge, the recognized "gold standard" for diagnosis of food allergy and some of the trials used open oral food challenges. The end point challenges varied in terms of the type of food used for challenge, with some testing the raw cow's milk or egg and some testing cooked egg for the case of egg immunotherapy. The volume of cow's milk or egg given in the final challenge varied.

Many children who underwent immune therapy were able to reach tolerance to cow's milk or egg in these studies however overall there was a high incidence of adverse reactions and a risk of loss of clinical desensitization after small periods off therapy [38]. Keet et al. reported loss of tolerance in as little as one week off SOTI [38]. The longest follow up period after the oral immunotherapy reported was three years for egg allergy [46] and four years for cow's milk allergy [56]. There are no safety data available testing the efficacy of oral immunotherapy beyond this length of time.

Many of the human intervention studies investigating clinical outcomes of individuals undergoing oral immunotherapy to cow's milk and egg have used skin prick testing as a marker of IgE levels or have measured serum specific IgE / IgG4 levels against the specific allergens to look for changes in the immune system towards tolerance (Tables 1 and 2). Only the more recent studies have begun to investigate changes at the cellular level $[38,46,49,53]$. Production of cytokines (IL10, TGFß and IFN $\gamma$ ) by T-cells influences the production of protective antibody responses by B cells including secretion of allergen-specific IgG4 and IgA and later inhibition of IgE. Immunotherapy has also been shown to lead to increases in protective $\operatorname{IgG}(1$ and 4 ) and $\operatorname{IgA}$, a decrease in $\operatorname{IgE}$ and a shift in the Th1/Th2 balance towards Th1, along with a decrease in T-cell proliferation and cytokine responses to allergens. Importantly there are increases in regulatory T-cells along with an increased production of IL-10 and TGFß implying that oral tolerance is being established [41].

Oral immunotherapy to egg and cow's milk shows potential as a management strategy for children with cow's milk and egg allergies and is able to induce desensitization and promote immune changes indicative of moves towards tolerance. However, SOTI carries a risk of adverse events both during the initiation phase and after periods off therapy, and the ideal treatment protocol is still to be identified. For researchers, a strategy that would facilitate this is the development of harmonized protocols, or, at least, harmonization of reporting of studies into SOTI to enable reporting, so that they can be clearly compared.

\section{Use of Baked Proteins for Oral Immunotherapy-An Alternative Approach?}

The form in which an oral allergen is delivered can influence the development of tolerance and the subsequent immune response profile generated. The studies summarized in Tables 1 and 2 all used raw proteins for the initial immunotherapy. If tolerance is achieved, in an individual, to raw uncooked proteins, then other forms of the food will also be tolerated. This has probably been the reason why centres have chosen to use raw foods as the vehicle for SOTI in the above reported clinical trials. Liquid or powders are also easy to measure, making the doses easy to deliver. However, SOTI using raw proteins is associated with a high rate of adverse events and often does not lead to permanent tolerance. 
It is recognized that some individuals with cow's milk and egg allergies are able to tolerate the proteins they are allergic to in the form of baked goods. Traditionally, allergic individuals were advised to avoid all forms of the allergenic protein in their diet, even if the individual was including the allergen in cooked food, for example, without any adverse events, in the belief that this could delay the resolution of the allergy [6]. However, it is now thought that achievement of tolerance to a heat treated protein may be the first step towards "outgrowing" the allergy and inducing tolerance [10]. In a longitudinal study of British children [60], tolerance to well-cooked egg (baked in a sponge cake) was gained at a median of 67 months (5.7 years) compared to 127 months (10.6 years) for raw egg. Resolution still continued to occur to cooked egg up to the age of 158 months (13.2 years) and for raw egg up to 182 months (15.2 years). There are no similar longitudinal studies that assess the natural history of resolution to products containing baked milk proteins; however, Wood et al. [61] recently published data on a multicentre cohort of children with cow's milk allergy. Of 293 participants, the median age of resolution of cow's milk allergy was 63 months (5.25 years). Of the 155 children with unresolved allergy, $32(20.6 \%)$ were able to tolerate products containing baked milk at the five year time point.

\section{Why Are Baked Proteins Different?}

Inclusion of baked milk or baked egg in the diet enables many foods that were previously avoided to be included in the diet, and this liberalization results in an improved quality of life for the child with food allergies. It is possible that regular inclusion of products containing the baked protein reduces the risk of reactions, due to accidental exposure to products containing egg or milk.

Proteins are three-dimensional molecules held together by electrostatic charge. IgE binding sites (or epitopes) may be sequential (several amino acids in a row) or conformational (part of the shape of the protein) [62]. Differences in allergenicity are, in part, due to changes in the structure of the proteins when heated, which affect the specific conformational IgE binding sites on the protein molecule. It is recognised that heat treatment of a protein can result in conformational changes in epitopes by affecting hydrogen bonds within the protein and, thus, affecting the ability of the IgE molecule to bind [63]. In the case of egg, heat treatment destroys the conformational epitopes that some individuals form IgE against, thus allowing ingestion of the egg without any adverse reaction. Some proteins are more susceptible to heat treatment than others, for example, the egg protein ovalbumin is more susceptible to heat treatment than ovomucoid [64]. In addition to altering the epitopes, heating the egg protein with wheat (for example, in a cake) forms a food matrix, also acting to reduce the allergenicity of the protein by affecting the digestibility of the proteins or making the $\operatorname{IgE}$ binding sites less accessible [64-66]. Other means of altering the allergenicity of a food protein include hydrolysis, acidification and other forms of food processing [67].

\section{Studies Assessing Tolerance to Baked Proteins in Individuals with Egg Allergy and Cow's Milk Allergy}

In 2008, Konstantinou [9] described the retrospective evaluation of 94 children (median age 24 months range $12-48$ months) with egg allergy $(n=55)$ or $\operatorname{IgE}$ sensitization to egg $(n=39)$. Ninety percent of the children tolerated an open oral food challenge to baked egg (containing $1.5 \mathrm{~g}$ baked egg 
protein). The 87 children who tolerated baked egg were then allowed to freely consume baked egg in their diet for six months and, then, were given a whole egg challenge. Only four out of 87 (4.6\%) children reacted to this challenge.

Lemon Mule et al. [8] were the first to study immune changes associated with consumption of baked egg in a wheat matrix by individuals with egg allergy. They enrolled 117 children with diagnosed egg allergy (mean age 6.6, range 1.6-18.6 years) and challenged them with heated (baked) egg in the form of a muffin or a waffle $-70 \%$ of the study group tolerated the baked egg and were then advised to include baked egg in their diet. The group was followed up at three, six and 12 months. Regular consumption of heated egg was associated with decreasing skin prick test (SPT) weal sizes to egg white and increasing ovalbumin- and ovomucoid-specific IgG4 levels. Intestinal permeability assessed by measurement of urinary clearance of non-metabolized sugars was no different between children consuming baked egg and those not consuming baked egg, and the children continued to grow well.

The study group was followed for six years, and the results of subsequent food challenges were reported by Nowak-Wegrzyn [68] and Leonard [69]. After incorporating heated egg into their diet, $58 \%$ of children eventually tolerated regular egg (French toast or scrambled egg) in their diets after a median of 16.6 months (interquartile range (IQR) 12.9-37.1 months). Children in the treatment group (consuming baked egg) were 14.6-times more likely to develop regular egg tolerance than children in the retrospective comparison group, and the children consuming baked egg developed tolerance earlier (median 50.0 vs. 78.7 months; $p<0.0001$ ) compared with those who did not.

Nowak-Wegrzyn et al. [70] challenged 100 cow's milk allergic children (average age 7.5 years, range 2.1-17.3 years) with cow's milk baked in waffles or muffins. Seventy-five percent of the cow's milk allergic children tolerated baked milk. Those who tolerated baked milk were asked to consume products containing baked milk at home for three months and, then, were re-evaluated. Immune response (serum-specific IgE and IgG4), growth and intestinal permeability were also monitored. After three months, children consuming baked milk products had significantly smaller SPT and higher casein-IgG4 compared with baseline. On repeat challenges with uncooked cow's milk, the group of children who tolerated heated cow's milk outgrew their milk allergy quicker than the group who did not tolerate heated cow's milk in their diet [7].

Kim [7] reported the results of the long-term follow-up of the group initially described by Nowak-Wegrzyn. The initial baked milk challenge was tolerated by 65 of 88 children. Compared with the children strictly avoiding milk, baked milk-tolerant children were more likely to become tolerant to unheated milk than baked milk-reactive children (Odds Ratio 2.8 (95\% Confidence Interval, 4.8-162.7); $p<0.01)$. No difference was noted in the milk-specific IgE levels between groups; however, both the casein $\operatorname{IgE}$ and $\beta$-lactoglobulin $\operatorname{IgE}$ values in the baked milk tolerant group decreased significantly over time. There was a significant increase in the median casein IgG4 value, but not the $\beta$-lactoglobulin IgG4 level from base line to final visit in the baked milk tolerant group. Eosinophilic esophagitis, which has been identified as a potential complication of SOTI [51], was reported in the baked milk study, but not the baked egg study. Two subjects in the active group and five in the comparison group had eosinophilic esophagitis, but this was not related to the intervention.

The baked egg and milk studies by Konstantinou, Lemon Mule et al. and Nowak-Wegrzyn et al. [8,9,70] are promising, but need substantiation, as the studies were not randomized controlled trials, and the 
results may be due to comparisons of different phenotypes of milk or egg allergy, changes with time or other unidentified confounders. Neither of the studies tested tolerance to the raw protein after a period of avoidance of the baked products, so it is unknown whether the effect was permanent.

There are several advantages of using baked products as a mode of inducing tolerance to egg or cow's milk protein. Once tolerance to a baked product is proven, the child is able to include it in their diet on a regular basis. Compared with traditional SOTI protocols, this means that there is less cost in terms of hospital admission times and inconvenience to the family, as there is no intense "up dosing" phase required. This approach is potentially safer compared with protocols, which rely on parents making up and administering the appropriate diluted dose of the allergen at home. The ability to consume baked products also moves the child towards a more normal, less restricted diet.

At present, there are no tests that predict tolerance to baked egg or baked milk proteins. Foods containing baked egg are reported to elicit anaphylaxis in egg allergic individuals, and as such, individuals chosen for this therapy should be carefully screened [9].

Furthermore, to date, there are few studies reporting immune mechanisms after oral immunotherapy, and this is one area that requires further research to help guide development of immunotherapy protocols. There is one study by Shreffler et al. that has shown a significantly higher percentage of proliferating casein-specific CD25/CD271 T-cells (regulatory T-cells involved in tolerance induction) from casein-induced peripheral blood mononuclear cell cultures taken from extensively heated milk-tolerant children compared with cells from extensively heated milk-reactive children. They showed no significant difference between the groups in the frequency of polyclonal T-cells or casein-specific effector T-cells. However, casein-specific regulatory T-cells correlated with the phenotype of having a mild transient milk allergy and favourable prognosis [36]. Induction of these regulatory T-cells is critical for the development or oral tolerance, and therefore, the study supports the concept of using heated allergens for tolerance induction.

While allergen specific oral immunotherapy has potential, there are significant questions regarding effectiveness and safety [71]. We have focused our review on tolerance induction to egg and milk; however, peanut allergy is also a significant health issue. Unfortunately, using heated peanut as a potential immunotherapeutic may not be feasible, as some forms of heat treatment (roasting) enables exposure of new epitopes to form, rendering this form of peanut more allergenic than boiled or fried peanut [72].

\section{Animal Studies Supporting the Use of Heated Proteins as Immunotherapeutic Agents}

There has been some work in animal models of allergy describing the effect on ingestion of heated egg proteins, but little about heated milk proteins. Martos [64] demonstrated that heat treatment of ovalbumin and ovomucoid reduces the allergenicity partly by changing the digestibility of the protein. Egg-sensitized $\mathrm{C} 3 \mathrm{H} / \mathrm{HeJ}$ mice were challenged with either untreated or heated ovalbumin or ovomucoid. Oral challenge with the unheated protein induced anaphylaxis in all of the mice, but not when challenged with the heated proteins. Even when challenged intraperitoneally, the majority of the mice only displayed mild reactions. In a separate experiment, mice fed heated ovalbumin had minimal T-cell proliferation in the mesenteric lymph nodes, or Peyer's Patches, compared with those consuming unheated ovalbumin, suggesting that heating ovalbumin affects the intestinal absorption of 
the intact form capable of triggering effector cells and T-cells. In this same paper, Martos [64] also reported that heated ovalbumin and ovomucoid still retained the ability to bind to serum from egg allergic children on immunoblotting, indicating that the epitopes were still intact.

Leonard [73] used a mouse model of SOTI to show that heated egg protein (ovomucoid) is able to desensitize egg allergic mice as effectively as unheated egg protein. $\mathrm{C} 3 \mathrm{H} / \mathrm{HeJ}$ mice sensitized to egg were treated with increasing doses of egg white or heated or native ovomucoid. Mice were challenged with ovalbumin or ovomucoid either one day or two weeks after the end of the desensitization period. At day 1, significantly fewer of the SOTI treated mice experienced anaphylaxis when compared with controls; however, when the mice were re-challenged at two weeks after ceasing SOTI, the previously desensitized mice were no longer protected from anaphylaxis. Mice given heated ovomucoid were completely protected against anaphylaxis compared with controls, as were mice given a higher dose egg white treatment compared with a low dose treatment. Antigen specific IL-13, IL-10 and IFN $\gamma$ responses were all significantly lower in SOTI treated mice compared with controls, and this difference remained two weeks post treatment. Whole genome microarray analysis of jejunum of sensitized control mice compared with those who were treated with SOTI indicated that administration of SOTI may lead to the regulation of a subset of genes in the intestine, including some expressed by intestinal epithelial cells. They postulated that the reduction in both the IL-10 and IL-13 after the SOTI indicated that the effect was related to an overall suppression rather than a shift in TH1/Th2 response.

As discussed, human studies investigating the ingestion of heated cow's milk and heated egg in allergic individuals demonstrated that this was associated with decreased skin test weal diameters and allergen-specific IgE levels and increased allergen-specific IgG4 levels [8,74]. Further studies are required to further quantify the immune changes that occur with ingestion of heated proteins to enable us to understand if ingestion of heated egg or heated cow's milk affects the natural history of egg and cow's milk allergies when compared with strict avoidance. Other important information that is required includes identification of diagnostic tests to accurately predict reactions to heat treated proteins and target potential candidates for this form of therapy, development of optimal protocols and evaluation of their efficacy and safety.

\section{Conclusions}

Oral induction of tolerance is a promising option for the management of food allergy, particularly with the increasing numbers of children being diagnosed with food allergies worldwide. However, SOTI with raw proteins is not free of risks, and there is much work to be done before it can be safely recommended as routine. The inclusion of baked cow's milk and egg proteins in the diet of children with IgE-mediated cow's milk and egg allergies appears to move the children towards a more tolerant immune profile, and the use of baked proteins is appealing, as it is safe, improves quality of life as the child moves towards a more normal diet and mimics the natural history of development of tolerance.

Areas for future research include unravelling the underlying mechanisms in the development of tolerance to a food. This will aid in the understanding and identification of the optimal route and protocols for dosing during the desensitization phase and also help to identify markers showing tolerance development as opposed to desensitization. Diagnostic tests to correctly identify candidates to approach regarding SOTI are required as are steps taken to optimize the safety of protocols. 
Harmonized study protocols or at least harmonization of reporting of studies into SOTI to enable reporting, so that they can be compared, is essential to the progression of this important area of research. Longer-term trials comparing children of like age groups and allergy phenotypes are required. Quality of life data should also be gathered from the individuals undergoing SOTI, as this has been largely absent from the studies reported, so far. Much more research is required to investigate if this will help children outgrow their allergies quicker, thus lessening the burden of managing food allergic disease.

\section{Acknowledgments}

MN is supported by an MS McLeod Research Trust PhD Scholarship. MM is an NHMRC Senior Research Fellow.

\section{Conflict of Interest}

$\mathrm{MN}$ and MG serve on a scientific advisory board for Nutricia. MM serves on scientific advisory boards for Nestle, Fonterra and Nutricia. Associated honoraria are paid to the institution to support conference travel and continuing education for post-graduate students and early career researchers. No other disclosures were reported.

\section{References}

1. WAO. White Book on Allergy; World Allergy Association: Milwaukee, WI, USA, 2011.

2. Sicherer, S.H.; Sampson, H.A. 9. Food allergy. J. Allergy Clin. Immunol. 2006, 117, S470-S475.

3. Sicherer, S.H.; Noone, S.A.; Munoz-Furlong, A. The impact of childhood food allergy on quality of life. Ann. Allergy Asthma Immunol. 2001, 2001, 461-464.

4. Skripak, J.; Matsui, E.; Mudd, K.; Wood, R. The natural history of IgE-mediated cow's milk allergy. J. Allergy Clin. Immunol. 2007, 120, 1172-1177.

5. Savage, J.; Limb, S.; Brereton, N.; Wood, R. The natural history of peanut allergy: Extending our knowledge beyond childhood. J. Allergy Clin. Immunol. 2007, 120, 717-719.

6. Allen, C.W.; Campbell, D.E.; Kemp, A.S. Food allergy: Is strict avoidance the only answer? Pediatr. Allergy Immunol. 2008, 20, 415-422.

7. Kim, J.S.; Nowak-Wegrzyn, A.; Noone, S.; Bencharitiwong, R.; Bloom, K.A.; Sampson, H.A. Tolerance to extensively heated milk $(\mathrm{hm})$ in children with cow's milk allergy: A follow up. J. Allergy Clin. Immunol. 2011, 127, AB27.

8. Lemon-Mulé, H.; Sampson, H.A.; Sicherer, S.H.; Shreffler, W.G.; Noone, S.; Nowak-Wegrzyn, A. Immunologic changes in children with egg allergy ingesting extensively heated egg. J. Allergy Clin. Immunol. 2008, 122, 977-983.e1.

9. Konstantinou, G.N.; Giavi, S.; Kalobatsou, A.; Vassilopoulou, E.; Douladiris, N.; Saxoni-Papageorgiou, P.; Papadopoulos, N.G. Consumption of heat-treated egg by children allergic or sensitized to egg can affect the natural course of egg allergy: Hypothesis-generating observations. J. Allergy Clin. Immunol. 2008, 122, 414-415. 
10. Clark, A.T.; Skypala, I.; Leech, S.C.; Ewan, P.W.; Dugué, P.; Brathwaite, N.; Huber, P.A.J.; Nasser, S.M. British society for allergy and clinical immunology guidelines for the management of egg allergy. Clin. Exp. Allergy 2010, 40, 1116-1129.

11. Konstantinou, G.N.; Kim, J.S. Paradigm shift in the management of milk and egg allergy: Baked milk and egg diet. Immunol. Allergy Clin. N. Am. 2012, 32, 151-164.

12. Nowak-Wegrzym, A.; Samson, H.A. Future therapies for food allergies. J. Allergy Clin. Immunol. 2011, 127, 558-573; quiz 574-575.

13. Jung, C.; Hugot, J.P.; Barreau, F. Peyer's patches: The immune sensors of the intestine. Int. J. Inflamm. 2010, 2010, 823710.

14. McGhee, J.R.; Fujihashi, K. Inside the mucosal immune system. PLoS Biol. 2012, 10, e1001397.

15. Niess, J.H.; Reinecker, H.C. Dendritic cells in the recognition of intestinal microbiota. Cell Microbiol. 2006, 8, 558-564.

16. Round, J.L.; O’Connell, R.M.; Mazmanian, S.K. Coordination of tolerogenic immune responses by the commensal microbiota. J. Autoimm. 2010, 34, J220-J225.

17. Castro-Sanchez, P.; Martin-Villa, J.M. Gut immune system and oral tolerance. Br. J. Nutr. 2013, 109, S3-S11.

18. Kim, J.S.; Sampson, H.A. Food allergy: A glimpse into the inner workings of gut immunology. Curr. Opin. Gastroenterol. 2012, 28, 99-103.

19. Smith, K.M.; Eaton, A.D.; Finlayson, L.M.; Garside, P. Oral tolerance. Am. J. Respir. Crit. Care Med. 2000, 162, S175-S178.

20. El-Merhibi, A.; Lymn, K.; Kanter, I.; Penttila, I.A. Early oral ovalbumin exposure during maternal milk feeding prevents spontaneous allergic sensitization in allergy-prone rat pups. Clin. Dev. Immunol. 2012, 2012, 396232.

21. Crabbe, P.A.; Nash, D.R.; Bazin, H.; Eyssen, H.; Heremans, J.F. Immunohistochemical observations on lymphoid tissues from conventional and germ-free mice. Lab. Invest. 1970, 22, 448-457.

22. Verhasselt, V.; Milcent, V.; Cazareth, J.; Kanda, A.; Fleury, S.; Dombrowicz, D.; Glaichenhaus, N.; Julia, V. Breast milk-mediated transfer of an antigen induces tolerance and protection from allergic asthma. Nat. Med. 2008, 14, 170-175.

23. Strobel, S. Immunity induced after a feed of antigen during early life: Oral tolerance $v$. Sensitisation. Proc. Nutr. Soc. 2001, 60, 437-442.

24. Strid, J.; Thomson, M.; Hourihane, J.; Kimber, I.; Strobel, S. A novel model of sensitization and oral tolerance to peanut protein. Immunology 2004, 113, 293-303.

25. Sudo, N.; Sawamura, S.; Tanaka, K.; Aiba, Y.; Kubo, C.; Koga, Y. The requirement of intestinal bacterial flora for the development of an IgE production system fully susceptible to oral tolerance induction. J. Immunol. 1997, 159, 1739-1745.

26. Weiner, H.L. Oral tolerance, an active immunologic process mediated by multiple mechanisms. J. Clin. Invest. 2000, 106, 935-937.

27. Tsuji, N.M.; Kosaka, A. Oral tolerance: Intestinal homeostasis and antigen-specific regulatory T-cells. Trends Immunol. 2008, 29, 532-540.

28. Mucida, D.; Kutchukhidze, N.; Erazo, A.; Russo, M.; Lafaille, J.J.; Curotto de Lafaille, M.A. Oral tolerance in the absence of naturally occurring tregs. J. Clin. Invest. 2005, 115, 1923-1933. 
29. Torgerson, T.R.; Ochs, H.D. Immune dysregulation, polyendocrinopathy, enteropathy, x-linked: Forkhead box protein 3 mutations and lack of regulatory T-cells. J. Allergy Clin. Immunol. 2007, 120, 744-750; quiz 751-752.

30. Bailey, M.; Haverson, K.; Inman, C.; Harris, C.; Jones, P.; Corfield, G.; Miller, B.; Stokes, C. The development of the mucosal immune system pre- and post-weaning: Balancing regulatory and effector function. Proc. Nutr. Soc. 2005, 64, 451-457.

31. Belz, G.; Heath, W.; Carbone, F. The role of dendritic cell subsets in selection between tolerance and immunity. Immunol. Cell Biol. 2002, 80, 463-468.

32. Torgerson, T.R.; Linane, A.; Moes, N.; Anover, S.; Mateo, V.; Rieux-Laucat, F.; Hermine, O.; Vijay, S.; Gambineri, E.; Cerf-Bensussan, N.; et al. Severe food allergy as a variant of IPEX syndrome caused by a deletion in a noncoding region of the foxp3 gene. Gastroenterology 2007, 132, 1705-1717.

33. Villablanca, E.J. Retinoic acid-producing des and gut-tropic foxp3 regulatory T-cells in the induction of oral tolerance. Oncoimmunology 2013, 2, e22987.

34. Nagatani, K.; Komagata, Y.; Asako, K.; Takayama, M.; Yamamoto, K. Antigen-specific regulatory T-cells are detected in peyer's patches after the interaction between T-cells and dendritic cells loaded with orally administered antigen. Immunobiology 2011, 216, 416-422.

35. Smith, M.; Tourigny, M.R.; Noakes, P.; Thornton, C.A.; Tulic, M.K.; Prescott, S.L. Children with egg allergy have evidence of reduced neonatal $\operatorname{cd} 4(+) \operatorname{cd} 25(+) \operatorname{cd} 127(\mathrm{lo} /-)$ regulatory $\mathrm{T}$-cell function. J. Allergy Clin. Immunol. 2008, 121, 1460-1466.

36. Shreffler, W.G.; Wanich, N.; Moloney, M.; Nowak-Wegrzyn, A.; Sampson, H.A. Association of allergen-specific regulatory T-cells with the onset of clinical tolerance to milk protein. J. Allergy Clin. Immunol. 2009, 123, 43-52.

37. Oppenheimer, J.J.; Nelson, H.S.; Bock, S.A.; Christensen, F.; Leung, D.Y. Treatment of peanut allergy with rush immunotherapy. J. Allergy Clin. Immunol. 1992, 90, 256-262.

38. Keet, C.A.; Frischmeyer-Guerrerio, P.A.; Thyagarajan, A.; Schroeder, J.T.; Hamilton, R.G.; Boden, S.; Steele, P.; Driggers, S.; Burks, A.W.; Wood, R.A. The safety and efficacy of sublingual and oral immunotherapy for milk allergy. J. Allergy Clin. Immunol. 2012, 129, 448-455.e5.

39. Barbi, E.; Longo, G.; Berti, I.; Matarazzo, L.; Rubert, L.; Saccari, A.; Lenisa, I.; Ronfani, L.; Radillo, O.; Ventura, A. Adverse effects during specific oral tolerance induction: In home phase. Allergol. Immunopathol. 2012, 40, 41-50.

40. Barbi, E.; Longo, G.; Berti, I.; Neri, E.; Saccari, A.; Rubert, L.; Matarazzo, L.; Montico, M.; Ventura, A. Adverse effects during specific oral tolerance induction: In-hospital "rush" phase. Eur. Ann. Allergy Clin. Immunol. 2012, 44, 18-25.

41. Wisniewski, J.; Agrawal, R.; Woodfolk, J.A. Mechanisms of tolerance induction in allergic disease: Integrating current and emerging concepts. Clin. Exp. Allergy 2013, 43, 164-176.

42. Yeung, J.P.; Kloda, L.A.; McDevitt, J.; Ben-Shoshan, M.; Alizadehfar, R. Oral immunotherapy for milk allergy. Cochrane Database Syst. Rev. 2012, doi:10.1002/14651858.CD009542.pub2.

43. Skripak, J.M.; Nash, S.D.; Rowley, H.; Brereton, N.H.; Oh, S.; Hamilton, R.G.; Matsui, E.C.; Burks, A.W.; Wood, R.A. A randomized, double-blind, placebo-controlled study of milk oral immunotherapy for cow's milk allergy. J. Allergy Clin. Immunol. 2008, 122, 1154-1160. 
44. Martorell, A.; De la Hoz, B.; Ibanez, M.D.; Bone, J.; Terrados, M.S.; Michavila, A.; Plaza, A.M.; Alonso, E.; Garde, J.; Nevot, S.; et al. Oral desenstization as a useful treatment in 2-year-old children with cow's milk allergy. Clin. Exp. Allergy 2011, 41, 1297-1304.

45. Meglio, P.; Giampietro, P.G.; Carello, R.; Gabriele, I.; Avitabile, S.; Galli, E. Oral food desensitization in children with IgE-mediated hen's egg allergy: A new protocol with raw hen's egg. Pediatr. Allergy Immunol. 2013, 24, 75-83.

46. Burks, A.W.; Jones, S.M.; Wood, R.A.; Fleischer, D.M.; Sicherer, S.H.; Lindblad, R.W.; Stablein, D.; Henning, A.K.; Vickery, B.P.; Liu, A.H.; et al. Oral immunotherapy for treatment of egg allergy in children. N. Engl. J. Med. 2012, 367, 233-243.

47. Dello Iacono, I.; Verga, M.C.; Tripodi, S. Oral immunotherapy for egg allergy in children. N. Engl. J. Med. 2012, 367, 1471; author reply 1472-1473.

48. Patriarca, G.; Nucera, E.; Pollastrini, E.; Roncallo, C.; De Pasquale, T.; Lombardo, C.; Pedone, C.; Gasbarrini, G.; Buonomo, A.; Schiavino, D. Oral specific desensitization in food-allergic children. Dig. Dis. Sci. 2007, 52, 1662-1672.

49. Fuentes-Aparicio, V.; Alonso-Lebrero, E.; Zapatero, L.; Infante, S.; Lorente, R.; Angeles Munoz-Fernandez, M.; Correa-Rocha, R. Oral immunotherapy in hen's egg-allergic children increases a hypo-proliferative subset of cd4+ T-cells that could constitute a marker of tolerance achievement. Pediatr. Allergy Immunol. 2012, 23, 648-653.

50. Patriarca, G.; Nucera, E.; Roncallo, C.; Pollastrini, E.; Bartolozzi, F.; De Pasquale, T.; Buonomo, A.; Gasbarrini, G.; Di Campli, C.; Schiavino, D. Oral desensitizing treatment in food allergy: Clinical and immunological results. Aliment. Pharmacol. Ther. 2003, 17, 459-465.

51. Buchanan, A.D.; Green, T.D.; Jones, S.M.; Scurlock, A.M.; Christie, L.; Althage, K.A.; Steele, P.H.; Pons, L.; Helm, R.M.; Lee, L.A.; et al. Egg oral immunotherapy in nonanaphylactic children with egg allergy. J. Allergy Clin. Immunol. 2007, 119, 199-205.

52. Itoh, N.; Itagaki, Y.; Kurihara, K. Rush specific oral tolerance induction in school-age children with severe egg allergy: One year follow up. Allergol. Int. 2010, 59, 43-51.

53. Garcia Rodriguez, R.; Urra, J.M.; Feo-Brito, F.; Galindo, P.A.; Borja, J.; Gomez, E.; Lara, P.; Guerra, F. Oral rush desensitization to egg: Efficacy and safety. Clin. Exp. Allergy 2011, 41, 1289-1296.

54. Urra, J.M.; Garcia Rodriguez, R.; Feo Brito, F.; Mur, P.; Guerra, F. Oral desensitization to egg enables cd4+foxp3+ cells to expand in egg-stimulated cells. J. Investig. Allergol. Clin. Immunol. 2012, 22, 71-73.

55. Meglio, P.; Bartone, E.; Plantamura, M.; Arabito, E.; Giampietro, P.G. A protocol for oral desensitization in children with IgE-mediated cow's milk allergy. Allergy 2004, 59, 980-987.

56. Meglio, P.; Giampietro, P.G.; Gianni, S.; Galli, E. Oral desensitization in children with immunoglobulin E-mediated cow's milk allergy-follow-up at $4 \mathrm{yr}$ and 8 months. Pediatr. Allergy Immunol. 2008, 19, 412-419.

57. Narisety, S.D.; Skripak, J.M.; Steele, P.; Hamilton, R.G.; Matsui, E.C.; Burks, A.W.; Wood, R.A. Open-label maintenance after milk oral immunotherapy for IgE-mediated cow's milk allergy. J. Allergy Clin. Immunol. 2009, 124, 610-612. 
58. Alvaro, M.; Giner, M.T.; Vazquez, M.; Lozano, J.; Dominguez, O.; Piquer, M.; Dias, M.; Jimenez, R.; Martin, M.A.; Alsina, L.; et al. Specific oral desensitization in children with IgE-mediated cow's milk allergy. Evolution in one year. Eur. J. Pediatr. 2012, 171, 1389-1395.

59. Bedoret, D.; Singh, A.K.; Shaw, V.; Hoyte, E.G.; Hamilton, R.; DeKruyff, R.H.; Schneider, L.C.; Nadeau, K.C.; Umetsu, D.T. Changes in antigen-specific T-cell number and function during oral desensitization in cow's milk allergy enabled with omalizumab. Mucosal Immunol. 2012, 5, 267-276.

60. Clark, A.; Islam, S.; King, Y.; Deighton, J.; Szun, S.; Anagnostou, K.; Ewan, P. A longitudinal study of resolution of allergy to well-cooked and uncooked egg. Clin. Exp. Allergy 2011, 41, 706-712.

61. Wood, R.A.; Sicherer, S.H.; Vickery, B.P.; Jones, S.M.; Liu, A.H.; Fleischer, D.M.; Henning, A.K.; Mayer, L.; Burks, A.W.; Grishin, A.; et al. The natural history of milk allergy in an observational cohort. J. Allergy Clin. Immunol. 2013, 131, 805-812.

62. Alessandri, C.; Zennaro, D.; Zaffiro, A.; Mari, A. Molecular allergology approach to allergic diseases in the paediatric age. Ital. J. Pediatr. 2009, 35, 29-41.

63. Tey, D.; Heine, R.G. Egg allergy in childhood: An update. Curr. Opin. Allergy Clin. Immunol. 2009, 9, 244-250.

64. Martos, G.; Lopez-Exposito, I.; Bencharitiwong, R.; Berin, M.C. Mechanisms underlying differential food allergy response to heated egg. J. Allergy Clin. Immunol. 2011, 127, 990-997.

65. Faeste, C.; Løvberg, K.; Lindvik, H.; Egaas, E. Extractability, stability, and allergenicity of egg white proteins in differently heat-processed foods. J. AOAC Int. 2007, 90, 427-436.

66. Huang, F.R.; Lemon-Mule, H.; Bencharitiwong, R.; Sampson, H.A.; Nowak-Wegrzyn, A.H. Effect of heating and food matrix on egg white (EW) protein allergenicity. J. Allergy Clin. Immunol. 2009, 1, S180.

67. Mine, Y.; Yang, M. Recent advances in the understanding of egg allergens: Basic, industrial, and clinical perspectives. J. Agric. Food Chem. 2008, 56, 4874-4900.

68. Nowak-Wegrzyn, A.H.; Leonard, S.A.; Bencharitiwong, R.; Noone, S.; Lemon-Mule, H.; Sampson, H.A. Follow-up of the heated egg (HE) diet trial. J. Allergy Clin. Immunol. 2011, 127, AB25.

69. Leonard, S.A.; Sampson, H.A.; Sicherer, S.H.; Noone, S.; Moshier, E.L.; Godbold, J.; Nowak-Wegrzyn, A. Dietary baked egg accelerates resolution of egg allergy in children. J. Allergy Clin. Immunol. 2012, 130, 473-480.e1.

70. Nowak-Wegrzyn, A.; Bloom, K.A.; Sicherer, S.H.; Shreffler, W.G.; Noone, S.; Wanich, N.; Sampson, H.A. Tolerance to extensively heated milk in children with cow's milk allergy. J. Allergy Clin. Immunol. 2008, 122, 342-347.

71. Nurmatov, U.; Venderbosch, I.; Devereux, G.; Simons, F.E.; Sheikh, A. Allergen-specific oral immunotherapy for peanut allergy. Cochrane Database Syst. Rev. 2012, doi:10.1002/14651858. CD009014.pub2.

72. Beyer, K.; Morrow, E.; Li, X.M.; Bardina, L.; Bannon, G.A.; Burks, A.W.; Sampson, H.A. Effects of cooking methods on peanut allergenicity. J. Allergy Clin. Immunol. 2001, 107, 1077-1081. 
73. Leonard, S.A.; Martos, G.; Wang, W.; Nowak-Wegrzyn, A.; Berin, M.C. Oral immunotherapy induces local protective mechanisms in the gastrointestinal mucosa. J. Allergy Clin. Immunol. 2012, 129, 1579-1587.e1.

74. Kim, J.S.; Nowak-Wegrzyn, A.; Sicherer, S.H.; Noone, S.; Moshier, E.L.; Sampson, H.A. Dietary baked milk accelerates the resolution of cow's milk allergy in children. J. Allergy Clin. Immunol. 2011, 128, 125-131.e2.

(C) 2013 by the authors; licensee MDPI, Basel, Switzerland. This article is an open access article distributed under the terms and conditions of the Creative Commons Attribution license (http://creativecommons.org/licenses/by/3.0/). 